\title{
Increased lipocalin-2 vs reduced oxytocin in relation with adiposity, atherogenicity and hematological indices in metabolic syndrome patients with and without prediabetes
}

\author{
Tofiq $\mathrm{KO}^{1,2}$, Bulatova $\mathrm{N}^{1}$, Kasabri $\mathrm{V}^{1}$, Suyagh $\mathrm{M}^{1}$, Halaseh $\mathrm{L}^{2}$, Alalawi $\mathrm{S}^{1 *}$ \\ *School of Pharmacy, University of Jordan, Amman, Jordan. hotice162@gmail.com
}

\begin{abstract}
OBJECTIVES: The neuropeptide hormone- Oxytocin (OXT) and glycoprotein Lipocalin-2 (LCN-2) are strongly associated with cardiometabolic risks of insulin resistance in metabolic syndrome (MetS) and prediabetes (preDM). METHODS: In a cross sectional design we aimed to compare and correlate plasma levels of OXT and LCN-2 and a set of clinical parameters, adiposity indices, atherogenicity indices, and hematological indices in 29 MetS/ preDM individuals and 29 non-diabetic MetS subjects vs 30 normoglycemic lean controls. Colorimetric enzymatic assays of biomarkers were procured.

RESULTS: LCN-2 concentration $(\mathrm{ng} / \mathrm{mL})$ increased significantly in MetS/preDM vs controls. Substantially in MetS recruits (both non-diabetic and pre-diabetics; $n=58$ ); OXT directly correlated with visceral adiposity in$\operatorname{dex}(\mathrm{VAI})$, non-HDL-C/HDL-C ratio, TC/HDL-C ratio, LDL-C/HDL-C ratio, lipid accumulation product (LAP), and atherogenicity index of plasma (AIP). Impressively, LCN-2 correlated proportionally with waist circumference (WC), red cell distribution width (RDW), neutrophils, and neutrophils to lymphocytes ratio (NLR), but inversely with lymphocytes in the 58 (non- and preDM) MetS participants.

CONCLUSIONS: These pronounced variations and correlations of OXT and LCN-2 emphasize their putative molecular roles in MetS and preDM pathophysiologies. Thus, OXT and LCN-2 can be surrogate prognostic/diagnostic tools for the MetS/preDM pharmacotherapy/prevention (Tab. 3, Fig. 1, Ref. 44). Text in PDF www.elis.sk. KEY WORDS: prediabetes, metabolic syndrome, adiposity indices, hematology indices, atherogenicity indices, oxytocin, lipocalin-2.
\end{abstract}

\section{Introduction}

Diabetes mellitus (DM) is a complex, chronic illness and is actually a group of metabolic disorders characterized by hyperglycemia. DM results from either defect in insulin secretion, action, or both (1). Abnormalities in fat, carbohydrate, and protein metabolism occur due to deficient effects of insulin on target tissues, the former result in chronic micro- and macrovascular complications (2). Prediabetes (preDM) is a state of failing of pancreatic beta cells to compensate for the high glucose level and/or insulin resistance, usually due to excessive body weight, abdominal or visceral obesity, metabolic syndrome (MetS), dyslipidemia, and hypertension. Individuals, whose blood glucose does not meet the criteria to be diagnosed as diabetes nor have normal glycemic level are named "prediabetes" (1). Prediabetes includes impaired fasting glucose (IFG) (fasting plasma glucose (FPG) $100 \mathrm{mg} / \mathrm{dL}$ to $125 \mathrm{mg} / \mathrm{dL}(5.6 \mathrm{mmol} / \mathrm{L}$ to $6.9 \mathrm{mmol} / \mathrm{L}))$, or impaired glucose tolerance (IGT) (2-h PG in the 75-g OGTT $140 \mathrm{mg} / \mathrm{dL}$ to $199 \mathrm{mg} /$

${ }^{{ }^{*} \text { School of Pharmacy, University of Jordan, Amman, Jordan, and }{ }^{2} \text { School }}$ of Medicine, University of Jordan, Amman, Jordan

Address for correspondence: V. Kasabri, School of Pharmacy, University of Jordan, Queen Rania Street, Amman 11942, Jordan.
$\mathrm{dL}(7.8 \mathrm{mmol} / \mathrm{L}$ to $11.0 \mathrm{mmol} / \mathrm{L})$ ), or HbA1C 5.7 to $6.4 \%$ (39 to 47 $\mathrm{mmol} / \mathrm{mol})$. Each component will increase the risk for DM and its complications. DM is one of the top global health priorities of the $21^{\text {st }}$ century that is affecting more people each year. The number of people with DM will increase to 628.6 million people by 2045 (3).

MetS is defined by a collection of interconnected biochemical, physiological, clinical and metabolic factors that all together will increase the likelihood of T2DM, atherosclerotic cardiovascular disease and, eventually, mortality. These pathophysiological changes can lead to dyslipidemia, hypertension, glucose intolerance, and prothrombotic state $(4,5)$. According to Alberti (6), based on international diabetes federation (IDF, 3) clinical criteria for the diagnosis of MetS are defined by having three or more out of five parameters of elevated waist circumference (WC) For men $\geq 94$ $\mathrm{cm}$, and for women $\geq 80 \mathrm{~cm}$, elevated fasting glucose $\geq 100 \mathrm{mg} / \mathrm{dL}$; or previously diagnosed T2DM, elevated blood pressure (Systolic $\geq 130 \mathrm{~mm} \mathrm{Hg}$ and/or diastolic $\geq 85 \mathrm{~mm} \mathrm{Hg}$ ), elevated triglyceride $\geq 150 \mathrm{mg} / \mathrm{dL}$, or specific treatment for this lipid abnormality, and reduced HDL-C For males $<40 \mathrm{mg} / \mathrm{dL}$, and for females $<50 \mathrm{mg} /$ $\mathrm{dL}$, or specific treatment for this lipid abnormality (6).

Oxytocin (OXT) has a nine amino-acid peptide structure that is similar to antidiuretic peptide-arginine vasopressin (AVP)(7). Both are neuropeptides produced by supraoptic nucleus (SON) 
and paraventricular nuclei (PVN) of hypothalamus and secreted by posterior pituitary (8). Oxytocin receptors (OXTR) are located centrally and peripherally and this is the reason behind OXT beneficial effect on CNS and its usefulness in obstetrics (9). Pregnant uterus is one of the traditional targets of OXT with its uterotonic effect used clinically to induce labor as it promotes contraction of the uterus and milk ejection during breast feeding (10). OXT receptors are upregulated during labor to 200 times more than in non-pregnant state (11). Clinical studies successfully proved that OXT nasal spray excellently induced weight loss and metabolic improvements in patients with obesity (12). Despite the basic researches that have been completed throughout the years for beneficial effects of OXT to treat obesity, using OXT will depend on our ability to integrate the diversity in its action between behavioral and metabolic to use it as an obesity treatment (13). Additionally, OXT was recently revealed to have several new roles in the cardiovascular system. In pathological conditions, the hormone exerts anti-inflammatory and cardioprotective properties, and improves vascular and metabolic functions. Thus, OXT has potential for therapeutic use (14-16). Of note, OXT supplementation alleviated age-related insulin resistance (IR) through down regulation of pro-inflammatory cytokine gene expression, further easing oxidative status in insulin -sensitive tissue without affecting body weight (17). OXT might be a potential therapeutic drug target in preventing pancreatic beta cell death (18).

Lipocalin-2 (LCN-2) is also known as neutrophil gelatinaseassociated lipocalin (NGAL). It is a $25-\mathrm{kDa}$ glycoprotein that was initially found in mouse kidney and human neutrophils (19). LCN-2 belongs to lipocalin family, a group of adipokines that have small hydrophobic molecules in circulation (19). It is secreted from adipocytes and its secretion and expression is upregulated by two pro-inflammatory cytokines in adipose tissue, interferon (IFN) gamma and tumor necrosis factor (TNF) alpha; it can enhance IR and obesity $(20,21)$. A more recent study showed an increased level of LCN-2 expression in obese women compared to the lean controls, as well as in T2DM and IGT in obese women compared to NGT obese women, indicating the independency of LCN-2 level for predicting IGT and T2DM in obese women (22). Thus, this representative biomarker can be used in clinical studies on population to link its relation with metabolic disease, obesity, and inflammation (23).

Our study was aimed to compare both OXT and LCN-2 plasma levels in normoglycemic and newly diagnosed preDM MetS patients versus healthy lean individuals. Therefore, to link both OXT and LCN-2 to metabolic derangements and anomalies, correlation studies were established with each of (Tab. 1):

- Adiposity indices, namely, WC, HC, CI, LAP, BAI, WHR and WHtR.

- Inflammatory hematological indices, namely PLR, NLR, MLR, and RDW.

- Atherogenicity indices, namely, total cholesterol-to-high density lipoprotein ratio (TC/HDL ratio), low density lipoprotein-to-high density lipoprotein ratio (LDL-C/HDL-C ratio), nonHDL-C/ HDL-C ratio and atherogenicity index of plasma (AIP $=\log _{10}$ $\{\mathrm{TG} /$ HDL-C ratio\}).

- Correlating OXT and LCN-2 levels to each other.

\section{Participants, materials and methods}

\section{Study design}

This cross -sectional study was meant to compare plasma levels of both OXT and LCN-2 in control group of 30 participants, who were apparently healthy, lean (BMI $<25 \mathrm{Kg} / \mathrm{m}^{2}$ ), and normoglycemic $(\mathrm{HbA} 1 \mathrm{c}<5.7 \%$, FBS $<100 \mathrm{mg} / \mathrm{dL})$, and two groups of overweight $\left(\mathrm{BMI}>25 \mathrm{~kg} / \mathrm{m}^{2}\right)$ or obese $\left(\mathrm{BMI}>30 \mathrm{~kg} / \mathrm{m}^{2}\right)$ drugnaive MetS subjects, as defined by Alberti (6). The cases group included 29 normoglycemic MetS and 29 pre-diabetes MetS (Fig. 1). Individuals with any of the following assessed candidates with

Tab. 1. Indices of anthropometric adiposity, atherogenicity and hematological used in this study.

\begin{tabular}{|c|c|c|}
\hline Measure & Clinical relevance and impact & Equations \\
\hline Waist-to-hip ratio (WHR) & WHO (26) & WHR $=$ waist circumference $(\mathrm{cm}) \div$ hip circumference $(\mathrm{cm})$ \\
\hline Waist-to-height ratio (WHtR) & Hsieh et al (27) & WHtR $=$ waist circumference $(\mathrm{cm}) \div$ height $(\mathrm{cm})$ \\
\hline Conicity index (CI) & Valdez (28) & $\mathrm{CI}=$ waist circumference $(\mathrm{cm}) \div 0.109 \sqrt{ }$ weight $(\mathrm{kg}) \div$ height $(\mathrm{m})$ \\
\hline Body adiposity index (BAI) & Bergman et al (29) & $\mathrm{BAI}=\left[\right.$ hip circumference $(\mathrm{cm}) /\left(\right.$ height $\left.\left.(\mathrm{m})^{1.5}\right)\right]-18$ \\
\hline Lipid accumulation product (LAP) & Kahn and Valdez (30) & $\begin{array}{l}\mathrm{LAP}=(\text { waist circumference }[\mathrm{cm}]-65) \times(\text { triglyceride concentration }[\mathrm{mM}]) \\
\text { for men } \\
\mathrm{LAP}=(\text { waist circumference }[\mathrm{cm}]-58) \times(\text { triglyceride concentration } \\
[\mathrm{mM}]) \text { for women }\end{array}$ \\
\hline Visceral adipocity index (VAI) & DeNino et al (31) & $\begin{array}{l}\text { VAI = waist circumference }(\mathrm{cm}) \div 39.68+(1.88 * \mathrm{BMI}) *(\text { triglyceride con- } \\
\text { centration } \div 1.03) *(1.31 \div \mathrm{HDL}-\mathrm{C}) \text { for male } \\
\text { VAI = waist circumference }(\mathrm{cm}) \div 36.58+(1.89 * \mathrm{BMI}) *(\text { triglyceride con- } \\
\text { centration } \div 0.81) *(1.52 \div \mathrm{HDL}-\mathrm{C}) \text { for female }\end{array}$ \\
\hline Atherogenic index of plasma (AIP) & Dobiášová (32) & $\mathrm{AIP}=\log _{10}$ (triglyceride concentration/HDL-C) \\
\hline $\begin{array}{l}\text { Total cholesterol/HDL-C (TC/HDL-C) ratio } \\
\text { LDL-C/HDL-C ratio }\end{array}$ & Ascaso et al (33) & $\begin{array}{l}\text { Total cholesterol/HDL-C }=\text { Total cholesterol } \div \text { HDL- } \mathrm{C} \\
\text { LDL-C/HDL-C }=\text { LDL-C } \div \text { HDL-C }\end{array}$ \\
\hline Non-HDL-C & Virani (34) & Non-HDL.C $=$ total cholesterol-HDL.C \\
\hline Platelet-to lymphocyte ratio (PLR) & Mete Ural (35) & PLR $=$ Platelets $\div$ Lymphocytes \\
\hline Neutrophil-to-lymphocyte ratio (NLR) & Mete Ural (35) & NLR $=$ Neutrophils $\div$ Lymphocytes \\
\hline Monocyte-to-lymphocyte ratio (MLR) & Ji et al (36) & MLR $=$ Monocytes $\div$ Lymphocytes \\
\hline
\end{tabular}




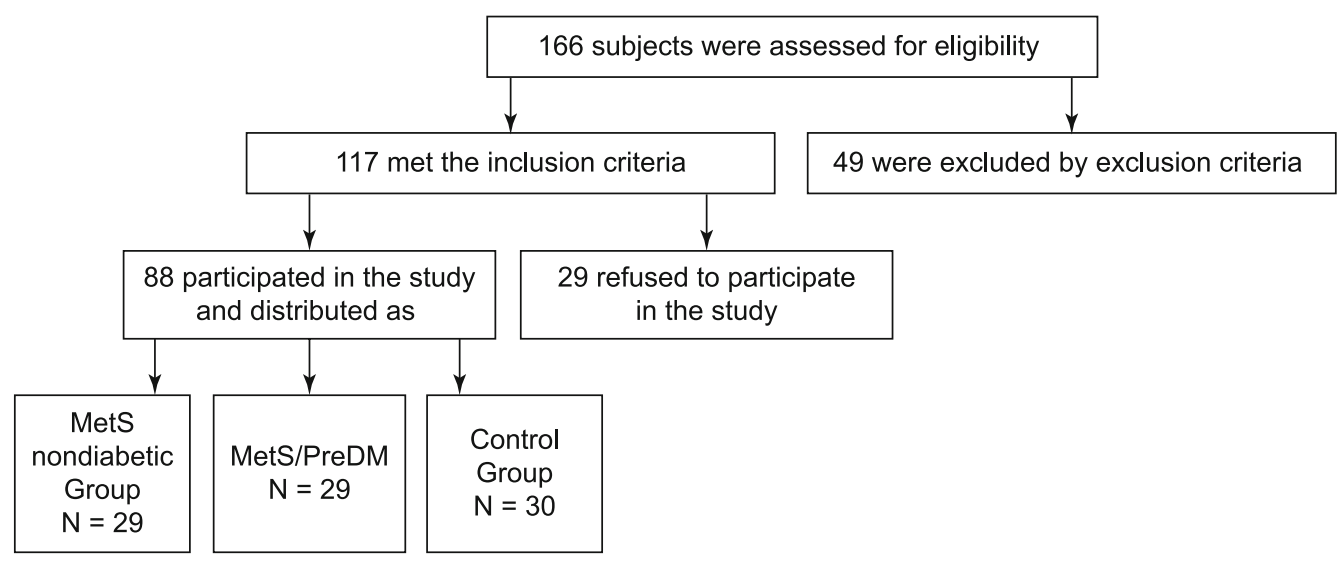

Fig. 1. Recruitment flow chart.

these criteria were excluded from the study:

- Non-fasting individuals.

- Any woman, who was pregnant or breast feeding.

- Clinical evidence of autoimmune or life-threatening disease (alcohol/drug abuse/recently diagnosed and untreated an endocrine disorder.

- Individuals with known inflammatory diseases, such as the bowel inflammatory disease.

- Obesity secondary to endocrine derangement other than DM.

- Any prior treatment with any kind of antidiabetic medications used for diabetes or any other medical condition.

\section{Study sample size}

Sample size was calculated by the formula of Wang and Chow (24):

$\mathrm{n}=2 * \mathrm{SD}^{2}\left(\mathrm{Z}_{\alpha}+\mathrm{Z}_{\beta}\right)^{2} / \Delta^{2}$

Where:

$\mathrm{N}$ : Sample size.

$Z_{\alpha}:$ Type one error $=1.96$ when $\alpha=5 \%$.

$Z_{\beta}$ : Type two error $=1.28$ when $\beta=10 \%$.

$\mathrm{SD}=$ Standard deviation OXT baseline from Qian et al (25) study equals to $1.38 \mathrm{ng} / \mathrm{L}$ and from a study by Rashad et al (22) equals to $6.6 \mathrm{ng} / \mathrm{mL}$ for $\mathrm{LCN}-2$.

$\Delta=$ the difference yielded between OXT levels of diabetic / pre-diabetic group vs the control group post-3 months treatment was $2.07 \mathrm{ng} / \mathrm{L}$. and for LCN-2the difference between IGT and T2DM was $5.4 \mathrm{ng} / \mathrm{mL}$. Thus, by applying Wang and Chow (24) equation, the minimum required number of individuals per each arm is 9 patients for OXT and 31 patients for LCN-2.

\section{Clinical settings and duration}

The study was conducted at the Family Medicine Clinic and the General Laboratories of the University of Jordan Hospital (UoJH) after obtaining the approvals from the UoJH Institutional Review Board (IRB) and the Scientific Research Committee at the School of Pharmacy, the University of Jordan. A consent form written in Arabic was handed to eligible individuals, who accepted to be enrolled in the study and asked for their signa- ture. Furthermore, they were interviewed about their medical and family history alongside with reviewing their medical file to collect clinical information and laboratory data. Patient recruitment started at the beginning of July 2017 and ended by the beginning of December 2017.The anthropometric data such as: height, waist circumference, blood pressure, and BMI were measured using specific tools. A venous blood was drawn from each candidate after 12 hours fasting to assess the levels of fasting plasma glucose (FPG) and lipid profile. The biochemical analysis of complete blood count (CBC), fasting lipid profile (HDL-C, LDL-C, TG, and $\mathrm{TC}$ ), FPG, and $\mathrm{HbAlc}$ were performed for each participant. Table 1 displays the indices that were used in this study. Competitive binding ELISA for plasma levels of OXT and sandwich type ELISA for plasma levels of LCN-2 were procured and assayed according to manufacturers' instructions (MyBioSource Inc. and Abcam Inc., USA).

\section{Statistical analysis}

All study subjects were coded according to the study arm they belong to. Data were entered and analyzed via IBM SPSS statistics 22 (SPSS, Inc., USA). Gender differences among the groups were tested using Chi-square test. One Way Analysis of Variance (ANOVA) test was used for comparing continuous independent variables between the study groups. Spearman correlation was used for continuous variables that were not normally distributed (as assessed by Shapiro Wilk test for assessment of normality assumption) to assess the relationship between them in the pooled sample of MetS patients (normoglycemic MetS and MetS/preDM patients).

\section{Results}

\section{Demographic data}

Females constituted $73.9 \%$ while men $26.1 \%$ of the study population (Tab. 2). Age had significant variation between the study arms with a $\mathrm{p}<0.001$ (Tab. 2); specifically between both MetS groups (nondiabetic MetS and MetS/preDM) vs control group ( $\mathrm{p}$ $<0.001$ and $\mathrm{p}=0.043$; respectively). 
Tab. 2. Comparison of study clinical and anthropometric parameters, adiposity, atherogenecity and hematological indices as well as metabolic risk biomarkers.

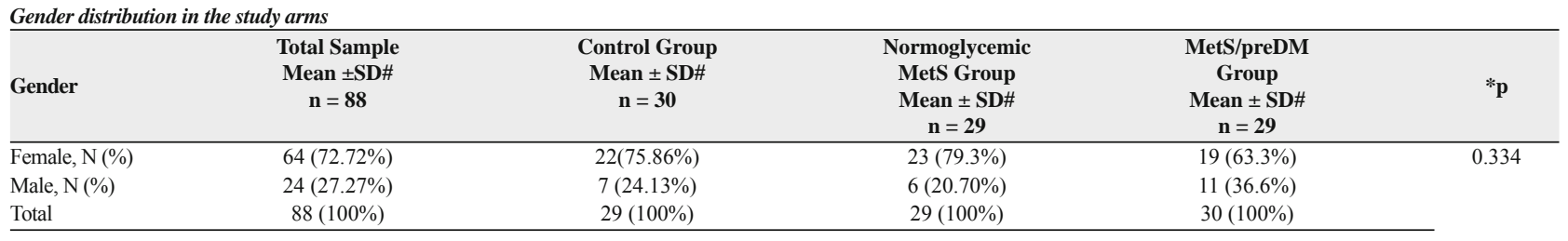

\begin{tabular}{|c|c|c|c|c|c|c|c|c|}
\hline \multicolumn{9}{|l|}{ Age comparison } \\
\hline & $\begin{array}{c}\text { Total Sample } \\
\text { Mean } \pm \text { SD\# } \\
\mathbf{n}=\mathbf{8 8}\end{array}$ & $\begin{array}{c}\text { Control Group } \\
\text { Mean } \pm \text { SD\# } \\
\mathbf{n}=\mathbf{3 0}\end{array}$ & $\begin{array}{c}\text { MetS Group } \\
\text { Mean } \pm \text { S.D\# } \\
\mathbf{n}=29\end{array}$ & $\begin{array}{c}\text { MetS/preDM } \\
\text { Group }\end{array}$ & p\#- & $\mathbf{p}$ & $\mathbf{p}$ & $\mathbf{p}$ \\
\hline Age (years) & $49.72 \pm 1.17$ & $44.39 \pm 2.05$ & $49.07 \pm 2.00$ & $55.68 \pm 1.51$ & $<0.001$ & 0.241 & $<0.001$ & 0.043 \\
\hline \multicolumn{9}{|l|}{ Clinical characteristics } \\
\hline & $\begin{array}{c}\text { Control Group } \\
\text { Mean } \pm \text { SD\# }\end{array}$ & \multicolumn{2}{|c|}{$\begin{array}{l}\text { Normoglycemic } \\
\text { MetS Group; }\end{array}$} & $\begin{array}{c}\text { MetS/preDM } \\
\text { Group }\end{array}$ & p\# & $\mathbf{p}^{1}$ & $\mathbf{p}^{2}$ & $\mathbf{p}^{3}$ \\
\hline & $\mathbf{n}=\mathbf{3 0}$ & Mean \pm & $n=29$ & Mean \pm SD\# $n=29$ & & & & \\
\hline$\overline{\mathrm{SBP}(\mathrm{mmHg})}$ & $117.1 \pm 2.23$ & \multicolumn{2}{|c|}{$138.17 \pm 1.64$} & $137.42 \pm 3.06$ & $<0.001$ & $<0.001$ & $<0.001$ & 1 \\
\hline $\mathrm{DBP}(\mathrm{mmHg})$ & $73.03 \pm 1.86$ & \multicolumn{2}{|c|}{$86.47 \pm 1.56$} & $85.42 \pm 2.26$ & $<0.001$ & $<0.001$ & $<0.001$ & 1 \\
\hline $\mathrm{FPG}(\mathrm{mg} / \mathrm{dL})$ & $85.31 \pm 1.40$ & \multicolumn{2}{|c|}{$88.53 \pm 1.72$} & $112.57 \pm 3.45$ & $<0.001$ & 1 & $<0.001$ & $<0.001$ \\
\hline $\mathrm{HbAlc} \%$ & $5.08 \pm 0.07$ & \multicolumn{2}{|c|}{$5.28 \pm 0.06$} & $6.22 \pm 0.13$ & $<0.001$ & 0.408 & $<0.001$ & $<0.001$ \\
\hline $\mathrm{TG}(\mathrm{mg} / \mathrm{dL})$ & $92.82 \pm 5.49$ & \multicolumn{2}{|c|}{$201.68 \pm 16.89$} & $210.93 \pm 25.64$ & $<0.001$ & $<0.001$ & $<0.001$ & 1 \\
\hline LDL-C (mg/dL) & $129.65 \pm 6.07$ & \multicolumn{2}{|c|}{$143.87 \pm 6.12$} & $142.21 \pm 7.23$ & 0.244 & 0.380 & 0.519 & 1 \\
\hline HDL-C (mg/dL) & $59.33 \pm 2.26$ & \multicolumn{2}{|c|}{$44.00 \pm 2.05$} & $47.19 \pm 3.07$ & $<0.001$ & $<0.001$ & 0.003 & 1 \\
\hline $\mathrm{TC}(\mathrm{mg} / \mathrm{dL})$ & $195.80 \pm 6.26$ & \multicolumn{2}{|c|}{$208.60 \pm 7.32$} & $218.03 \pm 8.40$ & 0.105 & 0.673 & 0.105 & 1 \\
\hline
\end{tabular}

\begin{tabular}{|c|c|c|c|c|c|c|c|}
\hline \multicolumn{8}{|c|}{ Adiposity indices } \\
\hline & \multirow{2}{*}{$\begin{array}{c}\text { Control Group } \\
\text { Mean } \pm \text { SD\# } \\
\mathbf{n}=30\end{array}$} & $\begin{array}{l}\text { Normoglycemic } \\
\text { MetS Group; }\end{array}$ & $\begin{array}{c}\text { MetS/preDM } \\
\text { Group }\end{array}$ & \multirow[t]{2}{*}{ p\# } & \multirow[t]{2}{*}{$\mathbf{p}^{1}$} & \multirow[t]{2}{*}{$\mathbf{p}^{2}$} & \multirow[t]{2}{*}{$\mathbf{p}^{3}$} \\
\hline & & Mean \pm SD\# $n=29$ & Mean \pm SD\# $n=29$ & & & & \\
\hline$\overline{\mathrm{WC}(\mathrm{cm})}$ & $87.23 \pm 1.81$ & $107.1 \pm 1.96$ & $107.66 \pm 2.30$ & $<0.001$ & $<0.001$ & $<0.001$ & 1 \\
\hline $\mathrm{HC}(\mathrm{cm})$ & $99.13 \pm 1.53$ & $116.73 \pm 2.00$ & $115.19 \pm 2.29$ & $<0.001$ & $<0.001$ & $<0.001$ & 1 \\
\hline $\mathrm{BMI}\left(\mathrm{Kg} / \mathrm{m}^{2}\right)$ & $23.20 \pm 0.34$ & $33.85 \pm 1.04$ & $33.10 \pm 1.23$ & $<0.001$ & $<0.001$ & $<0.001$ & 1 \\
\hline BAI & $29.92 \pm 0.78$ & $39.66 \pm 1.24$ & $38.77 \pm 1.71$ & $<0.001$ & $<0.001$ & $<0.001$ & 1 \\
\hline WHR & $0.88 \pm 0.01$ & $0.92 \pm 0.01$ & $0.94 \pm 0.01$ & 0.004 & 0.083 & 0.004 & 0.847 \\
\hline WHtR & $0.54 \pm 0.01$ & $0.67 \pm 0.01$ & $0.67 \pm 0.02$ & $<0.001$ & $<0.001$ & $<0.001$ & 1 \\
\hline C-index & $1.30 \pm 0.02$ & $1.34 \pm 0.01$ & $1.36 \pm 0.02$ & 0.059 & 0.424 & 0.057 & 1 \\
\hline LAP & $29.09 \pm 3.09$ & $108.99 \pm 10.79$ & $102.91 \pm 11.90$ & $<0.001$ & $<0.001$ & $<0.001$ & 1 \\
\hline VAI & $1.33 \pm 0.12$ & $3.95 \pm 0.45$ & $5.38 \pm 1.76$ & 0.026 & 0.252 & 0.024 & 1 \\
\hline
\end{tabular}

Atherogenicity indices

\begin{tabular}{|c|c|c|c|c|c|c|c|}
\hline & \multirow{2}{*}{$\begin{array}{c}\text { Control Group } \\
\text { Mean } \pm \text { SD\# } \\
n=30\end{array}$} & $\begin{array}{c}\text { Normoglycemic } \\
\text { MetS Group; }\end{array}$ & $\begin{array}{c}\text { MetS/preDM } \\
\text { Group }\end{array}$ & \multirow[t]{2}{*}{ p\# } & \multirow[t]{2}{*}{$\mathbf{p}^{1}$} & \multirow[t]{2}{*}{$\mathbf{p}^{2}$} & \multirow[t]{2}{*}{$\mathbf{p}^{3}$} \\
\hline & & Mean \pm S.D\# $\mathbf{n}=29$ & Mean \pm SD\# $n=29$ & & & & \\
\hline$\overline{\mathrm{AIP}}$ & $0.19 \pm 0.04$ & $0.63 \pm 0.05$ & $0.62 \pm 0.07$ & $<0.001$ & $<0.001$ & $<0.001$ & 1 \\
\hline LDL-C/HDL-C ratio & $2.40 \pm 0.17$ & $3.37 \pm 0.15$ & $3.59 \pm 0.43$ & 0.008 & 0.053 & 0.01 & 1 \\
\hline Non-HDL-C/HDL-C ratio & $2.48 \pm 0.20$ & $3.90 \pm 0.20$ & $5.16 \pm 1.32$ & 0.057 & 0.621 & 0.051 & 0.773 \\
\hline
\end{tabular}

\begin{tabular}{|c|c|c|c|c|c|c|c|}
\hline & \multirow{2}{*}{$\begin{array}{c}\text { Control Group } \\
\text { Mean } \pm \text { SD\# } \\
\mathbf{n}=\mathbf{3 0}\end{array}$} & $\begin{array}{l}\text { Normoglycemic } \\
\text { MetS Group }\end{array}$ & $\begin{array}{c}\text { MetS/preDM } \\
\text { Group }\end{array}$ & \multirow[t]{2}{*}{ p\# } & \multirow[t]{2}{*}{$\mathbf{p}^{1}$} & \multirow[t]{2}{*}{$\mathbf{p}^{2}$} & \multirow[t]{2}{*}{$\mathbf{p}^{3}$} \\
\hline & & Mean \pm S.D\# $\mathbf{n}=29$ & Mean \pm SD\#; $\quad n=29$ & & & & \\
\hline RDW & $14.4 \pm 0.23$ & $14.45 \pm 0.18$ & $14.62 \pm 0.25$ & 0.766 & 1 & 1 & 1 \\
\hline PLT count & $273.03 \pm 12.36$ & $274.50 \pm 11.42$ & $271.23 \pm 8.92$ & 0.978 & 1 & 1 & 1 \\
\hline Monocytes $\%$ & $5.63 \pm 0.28$ & $5.31 \pm 0.24$ & $5.40 \pm 0.24$ & 0.653 & 1 & 1 & 1 \\
\hline Neutrophiles\% & $57.39 \pm 1.12$ & $57.39 \pm 1.59$ & $59.82 \pm 1.72$ & 0.418 & 1 & 0.755 & 0.769 \\
\hline Lymphocytes\% & $33.49 \pm 1.17$ & $33.14 \pm 1.27$ & $30.20 \pm 1.46$ & 0.152 & 1 & 0.23 & 0.348 \\
\hline NLR & $1.81 \pm 0.10$ & $1.87 \pm 0.13$ & $2.53 \pm 0.43$ & 0.11 & 1 & 0.174 & 0.253 \\
\hline PLR & $8.46 \pm 0.49$ & $8.79 \pm 0.57$ & $10.33 \pm 1.08$ & 0.186 & 1 & 0.257 & 0.479 \\
\hline
\end{tabular}

\section{Molecular Metabolic risk biomarkers}

\begin{tabular}{|c|c|c|c|c|c|c|c|}
\hline$\overline{\mathrm{OXT}}(\mathrm{pg} / \mathrm{mL})^{\mathrm{b}}$ & $1776.27 \pm 130.62$ & $1759.77 \pm 223.87$ & $1400.18 \pm 154.08$ & 0.23 & 1 & 0.382 & 0.445 \\
\hline $\mathrm{LCN}-2(\mathrm{ng} / \mathrm{mL})^{\mathrm{b}}$ & $362.79 \pm 19.71$ & $429.41 \pm 20.13$ & $444.23 \pm 30.35$ & 0.044 & 0.165 & 0.049 & 1 \\
\hline \multicolumn{8}{|c|}{$\begin{array}{l}\text { Pairwise comparisons were done through Bonfferoni adjustment. \# adjusted mean and P obtained by ANOVA test. }{ }^{b} \text { Bootsrap results are based on } 1000 \text { bootsrap samples. } \\
\text { p }<0.05 \text { was highlighted bold. } p^{1} \text { normoglycemic MetS group versus control, } p^{2} \text { MetS/preDM versus control, }{ }^{3} \text { MetS/preDM versus normoglycemic MetS. (bolding refers to statistically significant finding } \\
\text { AIP: atherogenicity index of plasma, BAI: body adiposity index, C-index: conicity index, DBP: diastolic blood pressure, FPG: fasting plasma glucose, HbA1C\%: percent glycosylated- hemoglobi } \\
\text { HC: hip circumference, HDL-C: high density lipoprotein-cholesterol, LAP: lipid accumulation product, LDL-C/HDL-C: low density lipoprotein cholesterol to high density lipoprotein cholesterol rati } \\
\text { LDL-C: low density lipoprotein-cholesterol, MLR: monocyte-to-lymphocyte ratio, NLR: neutrophil-to-lymphocyte ratio, non-HDL-c/HDL: non high density lipoprotein to high density lipoprotein rati } \\
\text { Non-HDL-C: non-high density lipoprotein cholesterol, PLR: platelet-to-lymphocyte ratio. PLT: platelet, RDW: red cell width, SBP: systolic blood pressure, TC/HDL-C: total cholesterol to high densi } \\
\text { lipoprotein cholesterol ratio, TC: total cholesterol, TG: triglyceride, VAI: visceral adiposity index, WC: waist circumference, WHR: waist to hip ratio, WHtR: waist to height ratio. }\end{array}$} \\
\hline
\end{tabular}


Clinical, adiposity, atherogenicity, and hematological indices

Table 2 describes the clinical traits of each study arm. Both normoglycemic MetS and MetS-preDM groups had significantly higher values than the control group (except for nondiabetic MetS FPG and $\mathrm{HbA} 1 \mathrm{c})$. In addition, there was a significant difference between nondiabetic MetS and MetS/preDM groups in both FPG $(\mathrm{p}<0.001)$ and HbA1c $(\mathrm{p}<0.001)$. A significant difference was seen between both normoglycemic MetS and MetS/preDM groups in comparison to lean controls for all adiposity indices $(\mathrm{p}<0.05)$. Exceptionally, WHR had a significantly higher value in MetS/preDM group compared to the controls $(\mathrm{p}<0.05)$. Notably, there were significantly higher AIP and LAP in both normoglycemic MetS and MetS/PreDM group compared to the control group $(\mathrm{p}<0.05)$. Meanwhile, both LDL-C/HDL-C ratio and VAI showed significant differences in MetS/preDM group in comparison to the control group $(\mathrm{p}<0.05)$. Interestingly, all hematologic parameters failed to report any significant variability evidence in all of the study arms $(p>0.05)$.

\section{OXT and LCN-2 levels and correlations}

Table 2 shows the differences between both OXT and LCN-2 levels among three study groups. A significantly higher value of
LCN-2 was found in MetS/preDM group compared to the lean controls $(p<0.05)$. Conversely, OXT level was higher (though not significant) in control lean group compared to both MetS groups. In MetS patients (both non-diabetic and pre-diabetics; $\mathrm{n}=58$ ); OXT was (but not LCN-2) directly correlated with TG, LDL-C, LAP, non-HDL-C/HDL-C ratio, TC/HDL-C ratio, LDLC/HDL-C ratio, VAI and AIP. Evidently, LCN-2 (but not OXT) was correlated directly with WC, RDW, neutrophils, and NLR, but inversely with lymphocytes in MetS patients $(n=58)$, both non-diabetic and pre-diabetics (Tab. 3). Neither biomarker could appreciably associate with nonHDL-C, CI, BAI, WC, HC, BMI, WHR, WHtR or each other (the same table).

\section{Discussion}

Several studies investigated the level of OXT in relation to diabetes and MetS (37a, b, c, d). Our study could not report such a comparable significance despite the gradual decrease of OXT level in the control group $(1776.27 \pm 130.62 \mathrm{pg} / \mathrm{mL})$ vs normoglycemic MetS $(1759.77 \pm 223.87 \mathrm{pg} / \mathrm{mL})$ and MetS/preDM $(1400.18 \pm$ $154.08 \mathrm{pg} / \mathrm{mL}), \mathrm{p}>0.05$. Yuan et al (37a) reported a significantly

Tab. 3. Correlations of OXT and LCN-2 levels with clinical parameters, adiposity, atherogenicity and hematological indices in MetS patients (normoglycemics and pre-diabetics) $(\mathbf{n}=\mathbf{5 8})$.

\begin{tabular}{|c|c|c|c|c|c|c|c|c|c|c|}
\hline & & $\begin{array}{c}\text { SBP } \\
(\mathrm{mmHg})\end{array}$ & $\begin{array}{c}\text { DBP } \\
(\mathrm{mmHg})\end{array}$ & $\begin{array}{c}\text { FPG } \\
(\mathrm{mg} / \mathrm{dL})\end{array}$ & HbA1c & $\begin{array}{c}\text { TG } \\
(\mathrm{mg} / \mathrm{dL})\end{array}$ & $\begin{array}{l}\text { LDL-C } \\
\text { (mg/dL) }\end{array}$ & $\begin{array}{l}\text { HDL-C } \\
(\mathrm{mg} / \mathrm{dL})\end{array}$ & $\begin{array}{c}\text { TC } \\
\text { (mg/dL) }\end{array}$ & $\begin{array}{c}\text { Non-HDL-C } \\
\text { (mg/dL) }\end{array}$ \\
\hline $\mathrm{LCN}-2$ (ng/mL) & $\mathrm{R}$ & -0.08 & -0.09 & 0.03 & 0.00 & 0.15 & 0.24 & -0.04 & -0.17 & -0.08 \\
\hline \multirow{2}{*}{ OXT (pg/mL) } & $\mathrm{R}$ & -0.19 & -0.06 & -0.16 & -0.21 & $0.32^{*}$ & $0.31^{*}$ & 0.04 & -0.25 & 0.14 \\
\hline & $\mathrm{P}$ & 0.15 & 0.65 & 0.25 & 0.12 & 0.01 & 0.02 & 0.78 & 0.06 & 0.29 \\
\hline
\end{tabular}

\begin{tabular}{|c|c|c|c|c|c|c|c|c|c|c|c|c|}
\hline & & $\begin{array}{l}\text { WC } \\
(\mathrm{cm})\end{array}$ & $\begin{array}{l}\text { HC } \\
(\mathrm{cm})\end{array}$ & C-index & $\begin{array}{c}\text { BMI } \\
\left(\mathrm{kg} / \mathrm{m}^{2}\right)\end{array}$ & BAI & WHR & WHtR & LAP & VAI & LCN-2 & OXT \\
\hline \multirow{2}{*}{ LCN-2 (ng/mL) } & $\mathrm{R}$ & $0.27^{*}$ & 0.20 & 0.00 & 0.25 & 0.21 & 0.07 & 0.22 & 0.24 & 0.19 & 1.00 & 0.00 \\
\hline & $\mathrm{P}$ & 0.04 & 0.12 & 0.97 & 0.05 & 0.12 & 0.60 & 0.09 & 0.07 & 0.15 & . & 0.99 \\
\hline \multirow{2}{*}{ OXT (pg/mL) } & $\mathrm{R}$ & -0.03 & 0.00 & 0.02 & -0.04 & 0.05 & 0.01 & 0.00 & 0.31 & 0.35 & 0.00 & 1.00 \\
\hline & $\mathrm{P}$ & 0.84 & 0.96 & 0.86 & 0.76 & 0.75 & 0.90 & 0.99 & 0.02 & 0.01 & 0.99 & . \\
\hline
\end{tabular}

Correlations of $\mathrm{OXT}$ and $\mathrm{LCN}-2$ with atherogenicity indices.

\begin{tabular}{llcccc}
\hline & & Non-HDL C/HDL-C ratio & TC/HDL-C ratio & LDL-C/HDL-C ratio & 0.11 \\
\multirow{2}{*}{$\mathrm{LCN}-2(\mathrm{ng} / \mathrm{mL})$} & $\mathrm{R}$ & 0.16 & 0.16 & 0.18 \\
& $\mathrm{P}$ & 0.23 & 0.23 & 0.40 & $\mathbf{0 . 2 8}$ \\
\hline \multirow{2}{*}{$\mathrm{OXT}(\mathrm{pg} / \mathrm{mL})$} & $\mathrm{R}$ & $\mathrm{0.32}$ & $\mathbf{0 . 3 2}$ & $\mathbf{0 . 0 4}$ \\
& $\mathrm{P}$ & $\mathbf{0 . 0 2}$ & $\mathbf{0 . 0 2}$ & $\mathbf{0 . 3 1}$ \\
\hline
\end{tabular}

Correlations of OXT and LCN-2 with hematological indices.

\begin{tabular}{llcccccccc}
\hline & & RDW & PLT Count & Monocyte\% & Neutrophil\% & Lymphocytes\% & MLR & NLR & PLR \\
\hline \multirow{2}{*}{ LCN-2 (ng/mL) } & R & $\mathbf{0 . 2 7}$ & -0.22 & -0.07 & $\mathbf{0 . 2 9}$ & $\mathbf{- 0 . 3 5}$ & 0.21 & 0.20 \\
& $\mathrm{P}$ & $\mathbf{0 . 0 4}$ & 0.09 & 0.63 & $\mathbf{0 . 0 3}$ & $\mathbf{0 . 0 0}$ & 0.12 \\
\hline \multirow{2}{*}{$\mathrm{OXT}(\mathrm{pg} / \mathrm{mL})$} & $\mathrm{R}$ & 0.04 & -0.02 & -0.03 & 0.02 & 0.08 & -0.06 & -0.02 \\
& $\mathrm{P}$ & 0.76 & 0.90 & 0.84 & 0.87 & 0.55 & 0.12 & -0.04 \\
\hline
\end{tabular}

Spearman correlation coefficient $r_{s}$ was used, $r_{s}=0.1-.0 .29$ small relationship, $r_{s}=0.3-0.49$ moderate relationship, $r_{s}>0.5$ high relationship. (bolding refers to statistically significant findings)

AIP: atherogenicity index of plasma, BAI: body adiposity index, C-index: conicity index, DBP: diastolic blood pressure, FPG: fasting blood glucose, HbA1C\%: percent glycosylated-hemoglobin, HC: hip circumference, HDL-C: high density lipoprotein-cholesterol, LAP: lipid accumulation product, LDL-C: low density lipoprotein-cholesterol, LDL-C/HDL-C: low density lipoprotein cholesterol-to-high density lipoprotein cholesterol ratio, MLR: monocyte-to-lymphocyte ratio, MPV: mean platelet volume, NLR: neutrophil-to-lymphocyte ratio, Non-HDL-C: non-high density lipoprotein-cholesterol, non-HDL-c/HDL: non-high density lipoprotein-to-high density lipoprotein ratio, OXT: oxytocin, PLR: platelet-to-lymphocyte ratio, PLT: platelet, RDW: red cell width, SBP: systolic blood pressure, TC: total cholesterol, TC/HDL-C: total cholesterol-tohigh density lipoprotein-cholesterol ratio, TG: triglycerides, VAI: visceral adiposity index, WC: waist circumference, WHR: waist-to-hip ratio, WHtR: waist to height ratio. 
lower level of OXT in newly diagnosed MetS subjects. MetS was identified according to IDF (3) criteria, while in our study we used criteria proposed by Alberti (6). Similar to our MetS/preDM patients, patients in the above study were newly diagnosed with diabetes and drug-naive. OXT level reported by Yuan et al (37a) for MetS was 8 (6.67-9.49) ng/L vs 9.07 (8.08-10.37) ng/L for non-MetS. For investigating OXT level in diabetic patients Qian et al (25) recruited subjects with NGT and newly diagnosed drugnaive T2DM. Then further classification was done into four subgroups depending on BMI, normal weight (NW) with NGT, obese with NGT, NW with T2DM, and obese with T2DM. Obesity was defined by WHO-Western Pacific Region diagnostic criteria (2). Diagnosis of T2DM was by WHO (2) diagnostic criteria vs ADA (1) criteria used in our study. OXT levels for the subgroups was as followed: NW with NGT 9.86 (8.77-12.06) ng/L, obese with NGT 9.23 (8.16-10.36) ng/L, NW with T2DM 7.89 (7.03-9.17) ng/L, and obese with T2DM 7.16(6.45-8.82) ng/L. A significantly lower OXT level was observed between NGT vs T2DM $(\mathrm{p}<0.01)$ and NW vs obese $(p<0.01)$. Neither one of these studies combined MetS status with preDM; the overlap of these conditions together may have affected the difference in our finding as compared to them (Tab. 2). Similar to our study, Al-Rawashdeh et al (38) investigated OXT level in non-diabetic MetS subjects with a sample size of 69 patients vs our 29; and 89 MetS-pre/T2DM patients vs our 29. OXT level was significantly lower in MetS-pre/T2DM (1222.46 \pm 514.55 $\mathrm{pg} / \mathrm{mL}$ ) compared to non-diabetic MetS participants (2323.42 \pm $848.68 \mathrm{pg} / \mathrm{mL})(\mathrm{p}<0.001)$. In a more recent study by Akour et al (39) to examine OXT level in 45 normoglycemic lean controls vs $190 \mathrm{MetS}$ patients (42 MetS only, $95 \mathrm{MetS} / \mathrm{preDM}$, and $53 \mathrm{MetS} /$ T2DM) drug naïve. OXT was significantly lower in both MetS groups (preDM and T2DM) compared to MetS-only group ( $\mathrm{p}<$ 0.001 ). Our study could not report such significant decrease like the two previous studies, mainly due to small sample size, nevertheless, reduced OXT level may be due to T2DM pathogenesis in MetS group while our study was exclusively targeting MetS/preDM population. Our study showed a positive correlation between OXT and TG $(r=0.32, p<0.05)$, LDL-C $(r=0.31, p<0.05)$, LAP $(r=$ $0.31, \mathrm{p}<0.05)$, VAI $(\mathrm{r}=0.035, \mathrm{p}<0.05$, non-HDL-C/HDL-C ratio $(\mathrm{r}=0.32, \mathrm{p}<0.06)$, TC/HDL-C ratio $(\mathrm{r}=0.32, \mathrm{p}<0.05)$, LDL-C/ HDL-C ratio $(r=0.28, p<0.05)$, and AIP $(r=0.31, p<0.02)($ Tab. 3$)$.

In contrast, Yuan et al (37a) reported that BMI $(\mathrm{r}=-0.172, \mathrm{p}<$ $0.05)$, WC $(\mathrm{r}=-0.231, \mathrm{p}<0.05), \mathrm{HbA1c}(\mathrm{r}=-0.427, \mathrm{p}<0.001)$, FPG $(r=-0.401, p<0.001)$, and TG $(r=-0.356, p<0.001)$ had an inverse relationship with OXT level in MetS subjects. Similarly in the study by Qian et al (25), an inverse correlation was seen in OXT level with BMI $(\mathrm{r}=-0.231, \mathrm{p}<0.01)$, WC $(\mathrm{r}=-0.205, \mathrm{p}<$ $0.01) \mathrm{HbA1c}(\mathrm{r}=-0.381, \mathrm{p}<0.01)$, FPG $(\mathrm{r}=-0.315, \mathrm{p}<0.01)$, TG $(r=-0.289, p<0.01)$, TC $(r=-0.223, p<0.01)$, and LDL-C $(\mathrm{r}=-0.181, \mathrm{p}<0.01)$ in T2DM and NGT population.

A significant increase in LCN-2 level was detected in several studies investigating patients with MetS and/or T2DM. In this study, LCN-2 level in MetS/pre-diabetic group was higher (444.23 $\pm 30.35 \mathrm{ng} / \mathrm{mL})$ compared to the control group $(362.79 \pm 19.71$ $\mathrm{ng} / \mathrm{mL})(\mathrm{p}<0.05)$ supporting previous data. Wang et al (19) conducted a study of four groups. The first group included diabetic subjects identified by fasting plasma glucose $\geq 7.0 \mathrm{mmol} / \mathrm{L}$, or $2-\mathrm{h}$ plasma glucose during a $75 \mathrm{~g}$ oral glucose tolerance test $\geq-11.1$ $\mathrm{mmol} / \mathrm{L}$, according to the WHO (2) criteria. The second group included MetS subjects defined according to National Cholesterol Education Program's Adult Treatment Panel III Report criteria. The third group was comprised of obese participants with a BMI of $\geq 30 \mathrm{~kg} / \mathrm{m}^{2}$, and the fourth group included lean participants with a BMI of $<25 \mathrm{~kg} / \mathrm{m}^{2}$. The study showed a significant increase in LCN-2 levels (mean (SD)) in obese, diabetic and MetS individuals: obese 105 (48.8) $\mu \mathrm{g} / \mathrm{L}$ vs lean 65.4 (33.7) $\mu \mathrm{g} / \mathrm{L}, \mathrm{p}=0.001$; diabetics 82.4 (43.6) $\mu \mathrm{g} / \mathrm{L}$ vs non-diabetics 69.6 (32.9) $\mu \mathrm{g} / \mathrm{L}(\mathrm{p}<0.05)$; MetS 83.2 (39.6) $\mu \mathrm{g} / \mathrm{L}$ vs no-MetS 67.5 (32.7) $\mu \mathrm{g} / \mathrm{L}(\mathrm{p}<0.05)$.

Furthermore, a study by Rashad et al (22) enrolled a study of obese women with BMI $\geq 30 \mathrm{~kg} / \mathrm{m}^{2}$ and 180 healthy lean controls women to investigate $\mathrm{LCN}-2$ serum levels as an early predictor of diabetes among obese women. This can be particularly related to our study since $73.9 \%$ of our participants were women. The obese women were then stratified into three subgroups according to their FPG based on ADA (1) into: normal glucose tolerance (NGT), intermediate glucose tolerance (IGT) and T2DM. Our finding was also consistent with the data of the above study, which revealed a significant gradual increase of LCN-2 from NGT to T2DM between the three obese women subgroups: NGT $76.8 \pm 9.2 \mathrm{ng} / \mathrm{mL}$, IGT $78.1 \pm 8.1 \mathrm{ng} / \mathrm{mL}$, and T2DM $83.5 \pm 6.6 \mathrm{ng} / \mathrm{mL}(\mathrm{p}<0.001$ compared to lean controls).

It was reported that both man and woman, whose age is between 37-75 years had higher levels of LCN-2 knowing that deficiency in LCN-2can induce lipid metabolism dysregulation and systemic alteration. Such systemic alteration can be increased with age (40). In our study, age was significantly higher in Mets/prediabetic group (55.68 \pm 1.509 years) vs the control group (44.39 \pm 2.051 years $)(p<0.001)$, which can explain significantly higher LCN-2 level in Mets/pre-diabetic group $(\mathrm{p}=0.049)$.

In Choi et al (41) study, serum LCN-2 level was significantly increased in patients with coronary heart disease (CHD) $(\mathrm{p}<$ $0.001)$. Moreover, LCN-2 correlated positively with weight $(\mathrm{r}=$ $0.26, \mathrm{p}<0.05)$ and negatively with HDL-C $(\mathrm{r}=-0.30, \mathrm{p}<0.05)$. Wang et al (19) reported a correlation of adiposity indices in total study population with LCN-2, namely, BMI $(\mathrm{r}=0.394, \mathrm{p}<0.001)$, $\mathrm{WC}(\mathrm{r}=0.404, \mathrm{p}<0.001)$, WHR $(\mathrm{r}=0.9, \mathrm{p}<0.001)$. Similar to this finding, both Rashad et al (22) and Chesnaye et al (40) found a significant correlation between LCN-2 with BMI $(r=0.205, \mathrm{p}<$ $0.05 ; \mathrm{r}=0.3862, \mathrm{p}<0.05$, respectively). Furthermore, we found that in a pool of MetS patients (both non-diabetic and preDM), LCN-2correlated with WC $(r=0.27, \mathrm{p}<0.05)$.

When comparing atherogenicity indices with LCN-2, Wang et al (19) reported a correlation in total study population with TG (r $=0.151, \mathrm{p}<0.05)$ and inverse correlation with HDL-C $(\mathrm{r}=-0.2$, $\mathrm{p}<0.05)$. The latter finding was supported by Choi et al (41) ( $\mathrm{r}=$ $-0.30, \mathrm{p}<0.05)$. Similar results were found by Rashad et al $(22)$ : for TG correlation $\mathrm{r}=0.2014(\mathrm{p}<0.05)$ and for HDL-C correlation $r=-0.299(p<0.001)$ and Chesnaye et al (40): for TG correlation $r=0.4811(p=0.01)$ and for HDL correlation $r=-0.4034$ $(p<0.05)$. Our study could not report such significant results in MetS pool of non-diabetics and pre-diabetics (TG: $r=0.15, p>$ 
762-769

0.05 , HDL-C: $\mathrm{r}=-0.04, \mathrm{p}>0.05$ ). We also found a positive correlation between $\mathrm{LCN}-2$ and hematological indices, namely, RDW $(\mathrm{r}=0.27, \mathrm{p}<0.05)$, neutrophils $(\mathrm{r}=0.29, \mathrm{p}<0.05)$ and NLR $(\mathrm{r}$ $=0.33, \mathrm{p}=0.01)$ and an inverse relationship with lymphocytes $(\mathrm{r}$ $=-0.35, \mathrm{p}<0.001)$.

\section{Conclusions}

LCN-2 levels were significantly higher in MetS pre-diabetic individuals vs healthy lean participants; (in general, exhibited descending pattern: MetS-preDM > normoglycemic MetS > controls).

Conversely, OXT levels exhibited a descending pattern in the study arms (MetS-preDM < normoglycemic MetS < controls); despite not achieving a statistical significance.

Normoglycemic MetS and preDM-MetS individuals had higher adiposity parameters such (WC, HC, WHR, WHtR, BMI, BAI and LAP) and atherogenicity parameter (AIP) as compared to apparently healthy lean participants. For most of these parameters, the highest levels were obtained in preDM-MetS patients.

In general, higher OXT and lower LCN-2 levels were associated with a favorable metabolic picture (lipid profile, A1C, glucose level, BP). Thus, our findings support the importance of OXT and LCN-2 in MetS and preDM pathogenesis and their potential utilization in the development of promising therapeutic approaches of these diseases.

\section{Study limitations}

This is a cross-sectional study with a single time point determination; thus, no causality relationship could be concluded. It cannot be precisely speculated whether the biomarker itself caused the metabolic disturbances of prediabetes and/or MetS or the prediabetes and/or MetS caused the level change of biomarker.

The inclusion criteria for the MetS pre-diabetic patients may have inflicted a degree of limitation on its novel and significant outcomes. For future considerations; patients with T2DM, or even with uncontrolled diabetes as well as patients with diabetic complications maybe considered for more elaborate comparison.

The study was conducted at The University of Jordan Hospital. Suggestively, more referral healthcare centers may be as well included.

The need for invasive blood drawing to detect metabolic risk biomarkers; mostly this case is not much tolerated in geriatrics and pediatrics patients.

\section{References}

1. American Diabetes Association. Standards of medical care in diabetes. Diabetes Care 2017; 40 (1): 1-142.

2. World Health Organization (WHO; 1999); Department of Non-communicable Disease Surveillance Geneva, Definition, Diagnosis, and Classification of Diabetes Mellitus and its Complications. Available at http:// apps.who.int/iris/bitstream/handle/10665/66040/WHO_NCD_NCS_99. 2.pdf? sequence $=1 \&$ isAllowed $=y$. Last accessed April $17^{\text {th }} 2018$.
3. International Diabetes Federation (IDF; 2017), Diabetes Atlas eighth edition, 1-150.

4. Grundy SM, Cleeman JI, Daniels SR et al. Diagnosis and management of the metabolic syndrome: an American Heart Association/National Heart, Lung, and Blood Institute scientific statement. Circulation 2005; 112 (17): 2735-2752.

5. Wilson PWF, D’Agostino RB, Parise $\mathbf{H}$ et al. Metabolic syndrome as a precursor of cardiovascular disease and type 2 diabetes mellitus. Circulation 2005; 112 (20): 3066-3072.

6. Alberti KG. International Diabetes Federation Task Force on Epidemiology and Prevention; Hational Heart, Lung, and Blood Institute; American Heart Association; World Heart Federation; International Atherosclerosis Society; International Association for the Study of Obesity: Harmonizing the metabolic syndrome: a joint interim statement of the International Diabetes Federation Task Force on Epidemiology and Prevention; National Heart, Lung, and Blood Institute; American Heart Association; World Heart Federation; International Atherosclerosis Society; and International Association for the Study of Obesity. Circulation 2009; 120 (16): 1640-1645.

7. Murphy D, Si-Hoe SL, Brenner S, Venkatesh B. Something fishy in the rat brain: molecular genetics of the hypothalamo-neurohypophysial system. Bioessayes 1998; 20 (9): 741-749.

8. Acher R, Chauvet J. The neurohypophysial endocrine regulatory cascade: precursors, mediators, receptors, and effectors. Front Neuroendocrinol 1995; 16 (3): 237-289.

9. Gimple G, Fahrenholz F. The Oxytocin Receptor System: Structure, Function, and Regulation. Physiol Rev 2001; 18 (2): 629-683.

10. Manning M, Cheng L, Wa K, Stoev S et al. Advances in the design of selective antagonists, potential to colytics, and radioiodinated ligands for oxytocin receptors. Adv Exp Med Biol 1995; 395: 559-583.

11. Fuchs AR, Fuchs F. Endocrinology of human parturition: a review. Brit J Obst Gyn 1984; 91 (10): 948-967.

12. Cai D, Purkayastha S. A New Horizon: Oxytocin as a Novel Therapeutic Option for Obesity and Diabetes. Drug Discov Today Dis Mechan 2013; 10 (1-2): e63-e68.

13. Olszewski PK, Klockars A, Levine AS. Oxytocin and potential benefits for obesity treatment. Curr Opin Endocrinol Diabetes Obes 2017; 10: 1097 .

14. Gutkowska J, Jankowski M. Oxytocin revisited: its role in cardiovascular regulation. J Neuroendocrinol 2012; 24 (4): 599-608.

15. Szeto A, Rossetti MA, Mendez AJ et al. Oxytocin administration attenuates atherosclerosis and inflammation in Watanabe Hertitable Hyperlipidemic rabbits. Psychoneuroendocrinol 2013; 38 (5): 685-693.

16. Szylberg L, Michalski J, Maciejewska M, Marszalek A. Oxytocin and its role and effects-recent findings. Arch Perinat Med 2013; 19 (1): 43-49.

17. Abood AM, Alghamdi BS. Oxytocin supplementation alleviates agerelated insulin resistance through down regulation of pro-inflammatory cytokine gene expression. Biomed Res 2017; 28 (5): 2209-2215.

18. Watanabe S, Wei FY, Matsunaga T et al. Oxytocin Protects against Stress-Induced Cell Death in Murine Pancreatic $\beta$-Cells. Sci Rep 2016; 6: 25185 .

19. Wang Y, Lam KSL, Kraegen EW et al. Lipocalin-2 Is an Inflammatory Marker Closely Associated with Obesity, Insulin Resistance, and Hyperglycemia in Humans. Clin Chem 2007; 53 (1): 34-41. 
20. Hotamisligil GS, Shargill NS, Spiegelman BM. Adipose expression of tumor necrosis factor: direct role in obesity-linked insulin resistance. Science 1993; 259 (5091): 87-91.

21. Stephens JM, Lee J, Pilch PF. Tumor necrosis factor-induced insulin resistance in 3T3-L1 adipocytes is accompanied by a loss of insulin receptor substrate-1 and GLUT4 expression without a loss of insulin receptor-mediated signal transduction. J Biol Chem 1997; 272 (2): 971-976.

22. Rashad NM, El-Shal AS, Etewa RL, Wadea FM. Lipocalin-2 Expression and Serum Levels as Early Predictors of Type 2 Diabetes Mellitus in Obese Women. Int Union Biochem Mol Biol Life 2017; 69 (2): 88-97.

23. Eichelmann F, Rudovich N, Pfeiffer AF et al. Novel adipokines: methodological utility in human obesity research. Int J Obes 2017; 41 (6): 976-981.

24. Wang H, Chow S. Sample Size Calculations in clinical research. 2007; $1-451$.

25. Qian W, Zhu T, Tang B et al. Decreased Circulating Levels of Oxytocin in Obesity and Newly Diagnosed Type 2 Diabetic Patients. J Clin Endocrinol Metab 2014; 99 (12): 4683-4689.

26. World Health Organization (WHO; 2008), Waist Circumference and Waist-Hip Ratio: Report of a WHO Expert Consultation. Geneva, 1-31. http://apps.who.int/iris/bitstream/handle/10665/44583/9789241501491_ eng.pdf; jsessionid $=$ uence $=1$.

27. Hsieh SD, Yoshinaga H. Waist/height ratio as a simple and useful predictor of coronary heart disease risk factors in women. Intern Med 1995; 34 (12): 1147-1152.

28. Valdez R. A simple model-based index of abdominal adiposity. J Clin Epidemiol 1991; 44 (9): 955-956.

29. Bergman RN, Stefanovski D, Buchanan TA et al. A Better Index of Body Adiposity. Obesity 2011; 19 (5): 1083-1089.

30. Kahn HS, Valdez R. Metabolic risks identified by the combination of enlarged waist and elevated triacylglycerol concentration. Am J Clin Nutr 2003; 78: 928-934.

31. DeNino WF, Tchernof A, Dionne IJ et al. Contribution of abdominal adiposity to age-related differences in insulin sensitivity and plasma lipids in healthy non-obese women. Diabetes Care 2001; 24 (5): 925-932.

32. Dobiasova M. AIP - atherogenic index of plasma as a significant predictor of cardiovascular risk: from research to practice. Vnitr Lek 2006; 52 (1): 64-71.
33. Ascaso J, González Santos P, Hernández Mijares A et al. Management of dyslipidemia in the metabolic syndrome. Recommendations of the Spanish HDL Forum. Am J CardiovascDrugs. 2007; 7 (1): 39-58.

34. Virani SS. Non-HDL cholesterol as a metric of good quality of care: opportunities and challenges. Texas Heart Inst J 2011; 38 (2): 160-162.

35. Mete Ural U, Sehitoglu I, Bayoglu Tekin Y, KirSahin F. Neutrophil-to-lymphocyte and platelet-to-lymphocyte ratios in patients with endometrial hyperplasia and endometrial cancer. J Obstetr Gyn Res 2015; 41 (3): 445-448.

36. Ji H, Li Y, Fan Z et al. Monocyte/lymphocyte ratio predicts the severity of coronary artery disease: a syntax score assessment. BMC Cardiovasc Dis $2017 ; 17$ (1): $1-8$.

37. Yuan G, Qian W, Pan R et al. Reduced circulating oxytocin and HighMolecular-Weight adiponectin are risk factors for metabolic syndrome. Endocrine J 2016; 63 (7): 655-662.

38. Szulc P, Amri EZ, Varennes A et al. High serum oxytocin is associated with metabolic syndrome in older men - The MINOS study. Diabetes Res Clin Pract 2016; 122: 17-27.

39. Leng G, Sabatier N. Oxytocin - The Sweet Hormone? Trends Endocrinol Metab 2017; 28 (5): 365-376.

40. Spetter MS, Hallschmid M. Current findings on the role of oxytocin in the regulation of food intake. Physiol Behav 2017; 176: 31-39.

41. Al-Rawasdeh A, Kasabri V, Bulatova $\mathbf{N}$ et al. The correlation between plasma levels of oxytocin and betatrophin in non-diabetic and diabetic metabolic syndrome patients: A cross sectional study from Jordan. Diabetes MetS: Clin Res Rev 2017; 11 (1): 59-67.

42. Akour A, Kasabri V, Bulatova $\mathrm{N}$ et al. Association of Oxytocin with Glucose Intolerance and Inflammation Biomarkers in Metabolic Syndrome Patients with and without Prediabetes. Rev Diabet Stud 2018; 14 (4: 364 371.

43. Chesnaye EDL, Apolinar LM, Andal NOD et al. Gender differences in lipocalin-2 plasmatic levels are correlated with age and the triglyceride/high-density lipoprotein ratio in healthy individuals. Permanyer 2016; 152 (5): 612-617.

44. Choi KM, Lee JS, Kim EJ et al. Implication of lipocalin-2 and visfatin levels in patients with coronary heart disease. Eur J Endocrinol 2008; 158 (2): 203-207. 